CRYSTALLOGRAPHIC COMMUNICATIONS

ISSN 2056-9890

Received 19 July 2019

Accepted 14 August 2019

Edited by W. T. A. Harrison, University of Aberdeen, Scotland

Keywords: crystal structure; tetrazole derivative; DFT calculation; Hirshfeld surface analysis.

CCDC reference: 1947200

Supporting information: this article has supporting information at journals.iucr.org/e

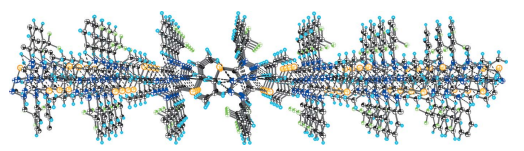

OPEN $\curvearrowright$ ACCESS

\section{Crystal structure, Hirshfeld surface analysis and computational studies of 5-[(prop-2-en-1-yl)sulfan- yl]-1-[2-(trifluoromethyl)phenyl]-1H-tetrazole}

\author{
Yurii Slyvka, ${ }^{a *}$ Evgeny Goreshnik, ${ }^{\mathrm{b}}$ Nazariy Pokhodylo ${ }^{\mathrm{a}}$ and Marian Mys'kiv ${ }^{\mathrm{a}}$ \\ ${ }^{\text {a}}$ Faculty of Chemistry, Ivan Franko National University of Lviv, Kyryla i Mefodia Str, 6, 79005 L'viv, Ukraine, and \\ ${ }^{\mathbf{b}}$ Department of Inorganic Chemistry and Technology, Jožef Stefan Institute, Jamova 39, SI-1000 Ljubljana, Slovenia. \\ *Correspondence e-mail: yurii.slyvka@Inu.edu.ua
}

The title compound, $\mathrm{C}_{11} \mathrm{H}_{9} \mathrm{~F}_{3} \mathrm{~N}_{4} \mathrm{~S}$, was synthesized from 2-(trifluoromethyl)aniline by a multi-step reaction. It crystallizes in the non-centrosymmetric space group $P n a 2_{1}$, with one molecule in the asymmetric unit, and is constructed from a pair of aromatic rings [2-(trifluoromethyl)phenyl and tetrazole], which are twisted by $76.8(1)^{\circ}$ relative to each other because of significant steric hindrance of the trifluoromethyl group at the ortho position of the benzene ring. In the crystal, very weak $\mathrm{C}-\mathrm{H} \cdots \mathrm{N}$ and $\mathrm{C}-\mathrm{H} \cdots \mathrm{F}$ hydrogen bonds and aromatic $\pi-\pi$ stacking interactions link the molecules into a three-dimensional network. To further analyse the intermolecular interactions, a Hirshfeld surface analysis, as well as interaction energy calculations, were performed.

\section{Chemical context}

Tetrazoles are a well-known class of aromatic five-membered heterocycles, which have been investigated since the end of the 19th century. Their biological properties, including antiviral, anticancer, anti-tuberculosis, antifungal and antioxidant activities have been shown by numerous studies (see, for example, Ostrovskii et al., 2017). They also are increasingly regarded as efficient and selective inhibitors of enzymes governing the metabolic processes in the human body (Pegklidou et al., 2010; Al-Hourani et al., 2012; Aggarwal et al., 2016).

Tetrazoles are well established as suitable precursors for the construction of other nitrogen-containing heterocycles such as pyrimidines (Shyyka et al., 2018; Pokhodylo et al., 2015), as well as being widely used as ligands in their own right to generate coordination compounds (Gaponik et al., 2006; Aromí et al., 2011). For example, allyl derivatives of $1 H$ tetrazole-5-thiols have been used for the preparation of copper(I) $\pi, \sigma$-complexes possessing non-linear optical properties (Slyvka et al., 2018, 2019). Among these, three copper(I) $\pi, \sigma$-coordination compounds, $\left[\mathrm{Cu}_{2}\left(\mathrm{C}_{11} \mathrm{H}_{9} \mathrm{~F}_{3} \mathrm{~N}_{4} \mathrm{~S}\right)_{2}\left(\mathrm{CF}_{3} \mathrm{SO}_{3}\right)_{2}\right]$ (Slyvka, 2015), $\left[\mathrm{Cu}\left(\mathrm{C}_{11} \mathrm{H}_{9} \mathrm{~F}_{3} \mathrm{~N}_{4} \mathrm{~S}\right)_{2}\right] \mathrm{BF}_{4}$ and $\left[\mathrm{Cu}\left(\mathrm{C}_{11} \mathrm{H}_{9} \mathrm{~F}_{3} \mathrm{~N}_{4} \mathrm{~S}\right.\right.$ )$\left.\left(\mathrm{NH}_{2} \mathrm{SO}_{3}\right)(\mathrm{MeOH})\right]$ based on 5-[(prop-2-en-1-yl)sulfanyl]-1[2-(trifluoromethyl)phenyl]-1H-tetrazole (I) $\left(\mathrm{C}_{11} \mathrm{H}_{9} \mathrm{~F}_{3} \mathrm{~N}_{4} \mathrm{~S}\right)$ have been reported recently (Slyvka et al., 2019). As part of our ongoing studies in this area, the synthesis and structure of the title compound, (I), are reported here.

\section{Structural commentary}

The title compound crystallizes in the non-centrosymmetric space group $\mathrm{Pna}_{1}$, with one molecule in the asymmetric unit. 
As shown in Fig. 1, it is constructed from two aromatic rings [2-(trifluoromethyl)phenyl and tetrazole rings], which are twisted relative to each other by $76.8(1)^{\circ}$ because of the significant steric hindrance of the trifluoromethyl group attached to $\mathrm{C} 10$. This dihedral angle is comparable with the analogous parameter in the same ligand when it is $\pi, \sigma$-coordinated to a copper atom in $\left[\mathrm{Cu}\left(\mathrm{C}_{11} \mathrm{H}_{9} \mathrm{~F}_{3} \mathrm{~N}_{4} \mathrm{~S}\right)_{2}\right] \mathrm{BF}_{4}$ [dihedral angle $\left.=78.0(1)^{\circ}\right]$ and $\left[\mathrm{Cu}\left(\mathrm{C}_{11} \mathrm{H}_{9} \mathrm{~F}_{3} \mathrm{~N}_{4} \mathrm{~S}\right)\left(\mathrm{NH}_{2} \mathrm{SO}_{3}\right)(\mathrm{MeOH})\right]$ [85.5 $\left.(1)^{\circ}\right]$ (Slyvka et al., 2019). The (prop-2-en-1-yl)sulfanyl group in (I) has an anticlinal conformation relative to the $\mathrm{C} 2-$ $\mathrm{C} 3$ bond and a synclinal conformation relative to the $\mathrm{S} 1-\mathrm{C} 2$ bond. The $\mathrm{S} 1-\mathrm{C} 2-\mathrm{C} 3-\mathrm{C} 4$ and $\mathrm{C} 1-\mathrm{S} 1-\mathrm{C} 2-\mathrm{C} 3$ torsion angles are $117.0(3)$ and $75.0(2)^{\circ}$, respectively.<smiles>C=CCSc1nnnn1-c1ccccc1C(F)(F)F</smiles>

\section{Supramolecular features}

As shown in Fig. 2 and listed in Table 1, the crystal structure of (I) features several weak intermolecular interactions. The hydrogen atoms of the (prop-2-en-1-yl)sulfanyl group are involved in $\mathrm{C}-\mathrm{H} \cdots \mathrm{N}$ bonding with the tetrazole ring of an adjacent molecule; these bonds link independent molecules into layers (Fig. 3). The layers are interconnected by $\mathrm{C}-$ $\mathrm{H} \cdots \mathrm{F}$ contacts into a three-dimensional network (Fig. 4).

\section{Hirshfeld surface analysis and computational study}

To further analyse the intermolecular interactions between the molecules of (I), Hirshfeld surface analysis through the mapping of the normalized contact distance $\left(d_{\text {norm }}\right)$ as well as

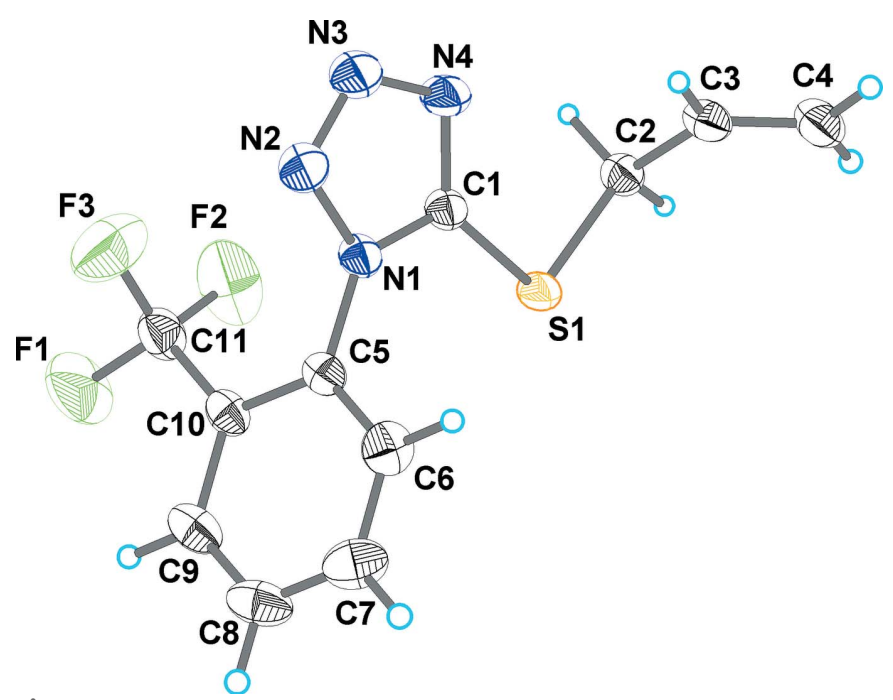

Figure 1

The molecular structure of (I) with displacement ellipsoids drawn at the $50 \%$ probability level.
Table 1

Hydrogen-bond geometry $\left(\AA{ }^{\circ}\right)$.

\begin{tabular}{lllll}
\hline$D-\mathrm{H} \cdots A$ & $D-\mathrm{H}$ & $\mathrm{H} \cdots A$ & $D \cdots A$ & $D-\mathrm{H} \cdots A$ \\
\hline $\mathrm{C} 2-\mathrm{H} 2 A \cdots \mathrm{N} 3^{\mathrm{i}}$ & 0.99 & 2.58 & $3.464(3)$ & 148 \\
$\mathrm{C} 2-\mathrm{H} 2 B \cdots \mathrm{N} 2^{\text {ii }}$ & 0.99 & 2.69 & $3.666(4)$ & 169 \\
$\mathrm{C} 3-\mathrm{H} 3 \cdots 3^{i i i}$ & 0.95 & 2.71 & $3.351(3)$ & 125 \\
$\mathrm{C} 4-\mathrm{H} 4 A \cdots \mathrm{N} 3^{\mathrm{i}}$ & 0.95 & 2.67 & $3.491(3)$ & 145 \\
$\mathrm{C} 4-\mathrm{H} 4 B \cdots \mathrm{F} 1^{\text {iv }}$ & 0.95 & 2.47 & $3.355(3)$ & 155 \\
$\mathrm{C} 6-\mathrm{H} 6 \cdots \mathrm{N} 4^{\text {iii }}$ & 0.95 & 2.76 & $3.601(3)$ & 148 \\
\hline
\end{tabular}

Symmetry codes: (i) $x+1, y, z$; (ii) $-x+1,-y+1, z-\frac{1}{2}$; (iii) $-x+1,-y+1, z+\frac{1}{2}$; (iv) $-x+\frac{3}{2}, y+\frac{1}{2}, z+\frac{1}{2}$.

calculation of the interaction energies were performed using CrystalExplorer (Turner et al., 2017; Spackman \& Jayatilaka, 2009). The most prominent interactions among the allyl group $\mathrm{H}$ atoms and tetrazole $\mathrm{N}$ atoms as well as among allylic $\mathrm{H}$ atoms and $\mathrm{F}$ atoms of neighbouring molecules can be seen in the Hirshfeld surface plot as the red areas (Fig. 5a). Fingerprint plots were produced to show the intermolecular surface bond distances with the regions highlighted for $\mathrm{C}-\mathrm{H} \cdots \mathrm{F}$

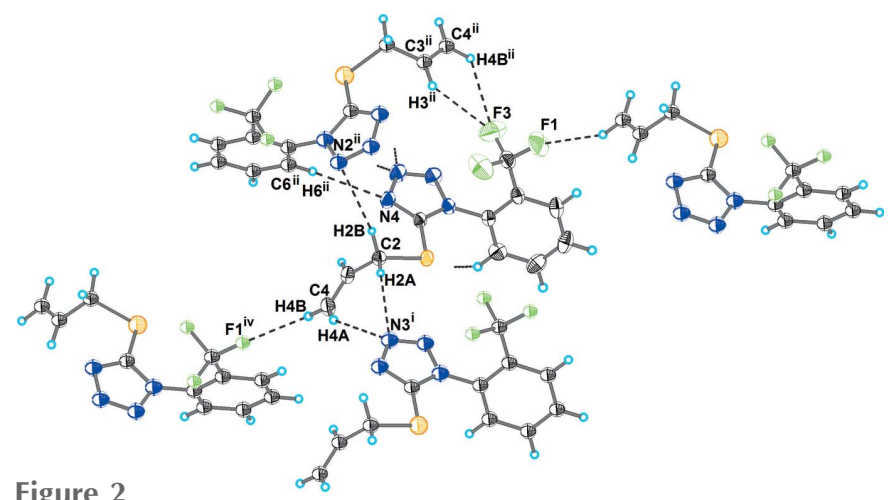

The hydrogen-bonding of molecules in (I). Hydrogen bonds are shown as dashed lines. The symmetry codes are as in Table 1.

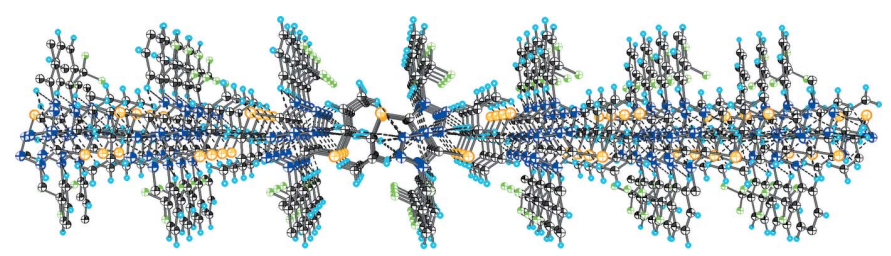

Figure 3

A C $-\mathrm{H} \cdots \mathrm{N}$-bonded layer in the structure of compound (I).

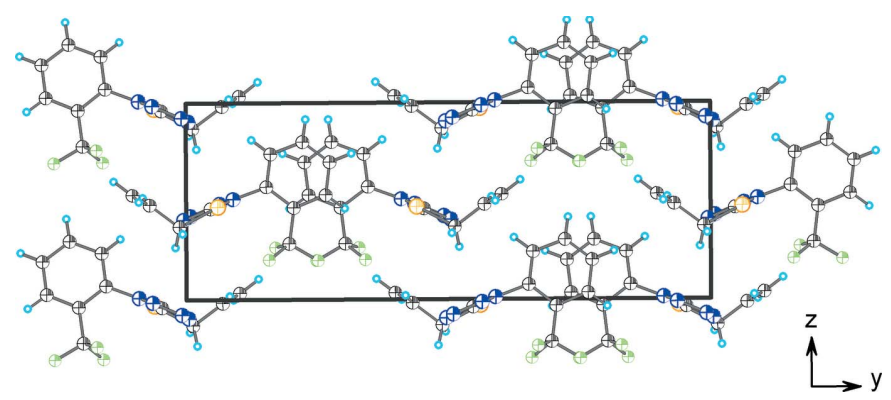

Figure 4

A view along the $a$ axis of the crystal packing of the title compound. 
Table 2

Interaction energies $\left(\mathrm{kJ} \mathrm{mol}^{-1}\right)$ for selected close contacts in the crystal of (I).

\begin{tabular}{|c|c|c|c|c|c|c|}
\hline Contact & $E_{\text {electrostatic }}$ & $E_{\text {polarization }}$ & $E_{\text {dispersion }}$ & $E_{\text {exchange-repulsion }}$ & $E_{\text {total }}$ & Symmetry operation \\
\hline $\mathrm{C} 4-\mathrm{H} 4 B \cdots \mathrm{F} 1$ & -1.4 & -0.3 & -5.2 & 5.7 & -2.6 & $-x+\frac{3}{2}, y+\frac{1}{2}, z+\frac{1}{2}$ \\
\hline $\mathrm{C} 4-\mathrm{H} 4 A \cdots \mathrm{N} 3 / \mathrm{C} 2-\mathrm{H} 2 A \cdots \mathrm{N} 3$ & -12.0 & -4.1 & -13.4 & 16.6 & -17.1 & $x+1, y, z$ \\
\hline $\mathrm{C} 2-\mathrm{H} 2 B \cdots \mathrm{N} 2 / \mathrm{C} 3-\mathrm{H} 3 \cdots \mathrm{F} 3 / \mathrm{C} 4-\mathrm{H} 4 B \cdots \mathrm{F} 3$ & -14.6 & -5.3 & -31.0 & 16.4 & -36.2 & $-x+1,-y+1, z-\frac{1}{2}$ \\
\hline$\left(\mathrm{CF}_{3} \mathrm{C}_{6} \mathrm{H}_{4}-\right) \cdots\left(\mathrm{CF}_{3} \mathrm{C}_{6} \mathrm{H}_{4}-\right)$ & -5.0 & -1.9 & -31.8 & 14.1 & -25.7 & $x-\frac{1}{2},-y+\frac{1}{2}, z$ \\
\hline
\end{tabular}

(Fig. 5b) and $\mathrm{C}-\mathrm{H} \cdots \mathrm{N}$ (Fig. 5c) interactions. The contribution to the surface area for $\mathrm{H} \cdot \mathrm{H}$. contacts is $19.8 \%$.

The interaction energies in (I) were calculated using a dispersion-corrected CE-B3LYP/6-31G(d,p) quantum level of theory, as available in CrystalExplorer. The total intermolecular energy is the sum of energies of four main components, viz. electrostatic, polarization, dispersion and exchange-repulsion factors of 1.057, 0.740, 0.871 and 0.618 , respectively (Mackenzie et al., 2017). The total calculated energy of the intermolecular interactions of (I) is $-115.9 \mathrm{~kJ} \mathrm{~mol}^{-}$. From Table 2, one can see the highest energy value $\left(-36.2 \mathrm{~kJ} \mathrm{~mol}^{-}\right)$covers $\mathrm{C}-\mathrm{H} \cdots \mathrm{N}$ and $\mathrm{C}-\mathrm{H} \cdots \mathrm{F}$ interactions with the neighbouring molecule generated by the symmetry code $-x+1,-y+1, z-\frac{1}{2}$. The interactions between the neighbouring 2-(trifluoromethyl)phenyl rings stacked along [100] cover $-25.7 \mathrm{~kJ} \mathrm{~mol}^{-1}$ and are mainly dispersive in nature.

\section{Database survey}

A survey of the Cambridge Structural Database (CSD version 5.39, last update August 2018; Groom et al., 2016) confirmed that 1-aryl substituted 5-[(prop-2-en-1-yl)sulfanyl]-1H-tetrazoles are known only as ligands in the structures of copper(I) and silver(I) $\pi$-complexes. In the crystal structures of bis $\left[\mu^{2}-\right.$ $\eta^{2}$-5-(allylsulfanyl)-1-phenyl- $1 H$-tetrazole]diaquadisilver bis(tetrafluoroborate) (refcode HAHTIV; Slyvka et al., 2011), bis $\left\{\mu-\eta^{2}-1\right.$-phenyl-5-[(prop-2-en-1-yl)sulfanyl]-1H-tetrazole $\}$ diaquadicopper bis(tetrafluoroborate) (JAHCON; Slyvka et al., 2010), bis $\left\{\mu-\eta^{2}-1-(4-c h l o r o p h e n y l)-5-[(\right.$ prop-2-en-1-yl)sulfanyl]-1 $H$-tetrazole diaquadicopper bis(tetrafluoroborate) ethanol solvate (JAHCUT; Slyvka et al., 2010) and bis $\{\mu-5-$ [(prop-2-en-1-yl)sulfanyl]-1-[2-(trifluoromethyl)phenyl]-1Htetrazole\}bis(trifluoromethanesulfonato)dicopper (JADHII; Slyvka, 2015), the tetrazole moieties are bonded to the metal ions through two heterocyclic nitrogen atoms and the allylic $\mathrm{C}=\mathrm{C}$ bond in the chelate-bridging mode. In catena- $\{(\mu$ sulfamato $)\left\{\eta^{2}-1\right.$-(3,5-dimethylphenyl)-5-[(prop-2-en-1-yl)sulfanyl]-1H-tetrazole\}copper(I)\} (ZEYRUT; Slyvka et al., 2018) (VI), the organic molecule is coordinated to the copper atom by the allylic $\mathrm{C}=\mathrm{C}$ bond and the only tetrazole nitrogen atom. As a result of the presence of back-donation from an occupied $3 d$ metal orbital to a low-lying empty $\pi^{*}$ orbital of the olefin, in all these compounds the double bond of the (prop-2-en-1yl)sulfanyl group is slightly elongated to $1.35-1.38 \AA$, in comparison with noncoordinated olefin bond value. The other S-substituted 1-phenyl-1H-tetrazole-5-thiol structures in the Cambridge Structural Database have different alkyl substituents, such as 2-naphthyl (TICRAY; Alves et al., 1996), 1,7,7trimethylbicyclo[2.2.1]hept-2-yl (GIJRAU; Bodrov et al., 2013) and benzoyl (BAZVAA; Kim et al., 2003).

\section{Synthesis and crystallization}

The title compound was synthesized from 2-(trifluoromethyl)aniline by a multi-step reaction. Commercially available 2-(trifluoromethyl)aniline $(1.611 \mathrm{~g}, 0.010 \mathrm{~mol})$ was dissolved in the minimum amount of benzene and treated with carbon

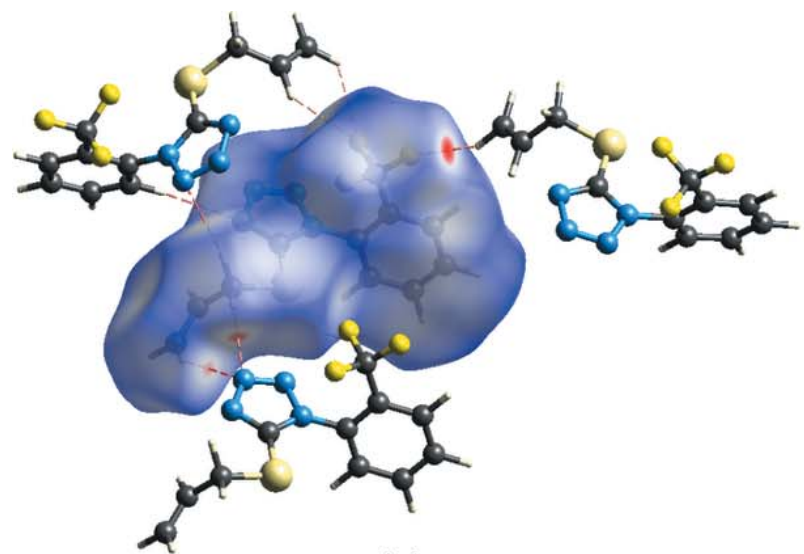

(a)

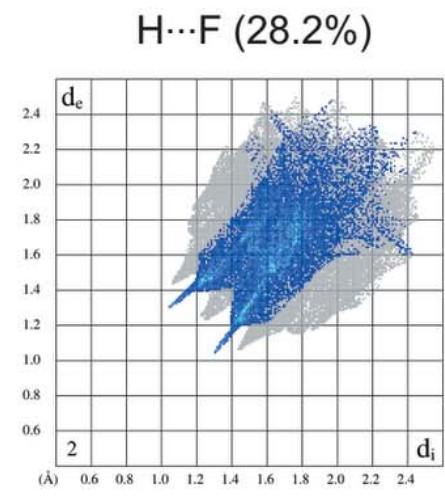

(b)
$\mathrm{H} \cdots \mathrm{N}(19.6 \%)$

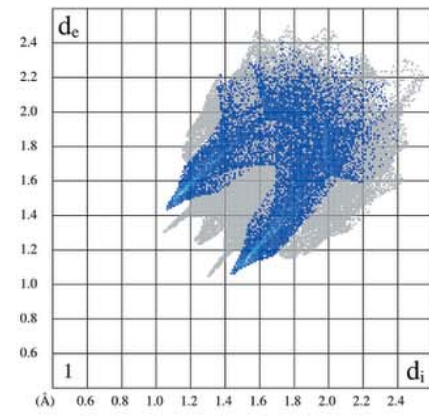

(c)

Figure 5

(a) Hirshfeld surface for molecule of (I) mapped with $d_{\text {norm }}$ over the range -0.15 to 1.2 showing $\mathrm{C}-\mathrm{H} \cdots \mathrm{N}$ and $\mathrm{C}-\mathrm{H} \cdots \mathrm{F}$ hydrogen-bonded contacts. Fingerprint plots for molecule resolved into $(b) \mathrm{F} \cdots \mathrm{H} / \mathrm{H} \cdots \mathrm{F}$ and $(c) \mathrm{N} \cdots \mathrm{H} / \mathrm{H} \cdots \mathrm{N}$ contacts. Neighbouring molecules associated with close contacts are also shown. 
disulfide $(0.7 \mathrm{ml}, \quad 0.01 \mathrm{~mol})$ and triethylamine $(1.4 \mathrm{ml}$, $0.010 \mathrm{~mol}$ ). The solution was cooled to $273 \mathrm{~K}$ and left for $5 \mathrm{~d}$. After complete precipitation of the triethylammonium dithiocarbamate salt, the solution was filtered. The solid was washed with anhydrous ether and air-dried for about $10 \mathrm{~min}$. The salt was then dissolved in about $7.5 \mathrm{ml}$ of chloroform, treated with $1.4 \mathrm{ml}$ of triethylamine and cooled to $273 \mathrm{~K}$. To this solution was added ethyl chloroformate $(1.02 \mathrm{ml}$, $0.01 \mathrm{~mol}$ ) dropwise over a $15 \mathrm{~min}$ period under intensive stirring. The resulting solution was stirred at $273 \mathrm{~K}$ for $10 \mathrm{~min}$ and allowed to warm to room temperature over $1 \mathrm{~h}$. The chloroform solution was washed with $3 \mathrm{M} \mathrm{HCI}$ and twice with water and dried over $\mathrm{Na}_{2} \mathrm{SO}_{4}$. The chloroform was evaporated and the 1-isothiocyanato-2-(trifluoromethyl)benzene was distilled in vacuo.

The obtained isothiocyanate $(1.016 \mathrm{~g}, 5.0 \mathrm{mmol})$ was mixed with water $(10 \mathrm{ml})$ and $\mathrm{NaN}_{3}(0.71 \mathrm{~g}, 0.011 \mathrm{~mol})$ and refluxed under intensive stirring until the suspension disappeared. The solution was cooled to room temperature and washed with TBME. The water fraction was separated and acidified with 3 $M \mathrm{HCl}$ (Caution! During the acidification beware of toxic $\mathrm{HN}_{3}$ gas). The sediment of 1-[2-(trifluoromethyl)phenyl]-1Htetrazole-5-thiol was separated by filtration and used for alkylation without further purification.

1-[2-(Trifluoromethyl)phenyl]- $1 H$-tetrazole-5-thiol $(0.985 \mathrm{~g}$, $0.004 \mathrm{~mol})$ was dissolved in a solution of $\mathrm{KOH}(0.22 \mathrm{~g}$, $0.004 \mathrm{~mol})$ in ethanol $(10 \mathrm{ml})$. To the solution allyl bromide ( $0.43 \mathrm{ml}, 0.005$ mole) was added and the mixture was heated at $323 \mathrm{~K}$ for $1 \mathrm{~h}$. The solvent was removed in vacuo and to the residue was added water $(5 \mathrm{ml})$ and dichloromethane $(10 \mathrm{ml})$. The dichloromethane was separated and removed to give the title compound. Colourless blocks of (I) were obtained by recrystallization from an ethanol solution, m.p. $336 \mathrm{~K}$.

NMR ${ }^{1} \mathrm{H}\left(400 \mathrm{MHz}\right.$, DMSO- $\left.d_{6}\right), \delta$, p.p.m. $8.03(d, J=7.3 \mathrm{~Hz}$, $\left.1 \mathrm{H}, \mathrm{H}_{\mathrm{Ph}}-3\right), 7.98-7.88\left(m, 2 \mathrm{H}, \mathrm{H}_{\mathrm{Ph}^{-}}-4,5\right), 7.71(d, J=7.3 \mathrm{~Hz}, 1 \mathrm{H}$, $\left.\mathrm{H}_{\mathrm{Ph}}-6\right), 5.94(t d, J=16.8,7.2 \mathrm{~Hz}, 1 \mathrm{H},=\mathrm{CH}), 5.36(d, J=$ $\left.16.8 \mathrm{~Hz}, 1 \mathrm{H},=\mathrm{CH}_{2}\right), 5.18\left(d, J=9.9 \mathrm{~Hz}, 1 \mathrm{H},=\mathrm{CH}_{2}\right), 3.98(d, J$ $\left.=6.9 \mathrm{~Hz}, 2 \mathrm{H}, \mathrm{CH}_{2}\right)$. Analysis calculated for $\mathrm{C}_{11} \mathrm{H}_{9} \mathrm{~F}_{3} \mathrm{~N}_{4} \mathrm{~S}: \mathrm{C}$, 46.15; H, 3.17; N, 19.57; S, 11.20; found: C, 45.97; H, 3.04; N, 19.49; S, 11.27.

\section{Refinement}

Crystal data, data collection and structure refinement details are summarized in Table 3. $\mathrm{H}$ atoms were positioned geometrically and refined using riding model, with $\mathrm{C}-\mathrm{H}=0.95$ or $0.99 \AA$ and $U_{\text {iso }}(\mathrm{H})=1.2 U_{\text {eq }}(\mathrm{C})$.

\section{Funding information}

EG gratefully acknowledges financial support from the Slovenian Research Agency (ARRS).

\section{References}

Aggarwal, S., Mahapatra, M. K., Kumar, R., Bhardwaj, T. R., Hartmann, R. W., Haupenthal, J. \& Kumar, M. (2016). Bioorg. Med. Chem. 24, 779-788.
Table 3

Experimental details.

\begin{tabular}{|c|c|}
\hline \multicolumn{2}{|l|}{ Crystal data } \\
\hline Chemical formula & $\mathrm{C}_{11} \mathrm{H}_{9} \mathrm{~F}_{3} \mathrm{~N}_{4} \mathrm{~S}$ \\
\hline$M_{\mathrm{r}}$ & 286.28 \\
\hline Crystal system, space group & Orthorhombic, Pna $2_{1}$ \\
\hline Temperature $(\mathrm{K})$ & 150 \\
\hline$a, b, c(\AA)$ & $7.6595(3), 20.9841(7), 7.8641$ (3) \\
\hline$V\left(\AA^{3}\right)$ & $1263.98(8)$ \\
\hline$Z$ & 4 \\
\hline Radiation type & Mo $K \alpha$ \\
\hline$\mu\left(\mathrm{mm}^{-1}\right)$ & 0.28 \\
\hline Crystal size $(\mathrm{mm})$ & $0.35 \times 0.24 \times 0.15$ \\
\hline \multicolumn{2}{|l|}{ Data collection } \\
\hline Diffractometer & $\begin{array}{l}\text { Rigaku Oxford Diffraction New } \\
\text { Gemini, Dual, Cu at home/near, } \\
\text { Atlas }\end{array}$ \\
\hline Absorption correction & $\begin{array}{l}\text { Analytical (CrysAlis PRO; Rigaku } \\
\text { OD, 2018) }\end{array}$ \\
\hline$T_{\min }, T_{\max }$ & $0.929,0.969$ \\
\hline $\begin{array}{l}\text { No. of measured, independent and } \\
\text { observed }[I>2 \sigma(I)] \text { reflections }\end{array}$ & $28170,3094,2730$ \\
\hline$R_{\text {int }}$ & 0.059 \\
\hline$(\sin \theta / \lambda)_{\max }\left(\AA^{-1}\right)$ & 0.680 \\
\hline \multicolumn{2}{|l|}{ Refinement } \\
\hline$R\left[F^{2}>2 \sigma\left(F^{2}\right)\right], w R\left(F^{2}\right), S$ & $0.035,0.079,1.07$ \\
\hline No. of reflections & 3094 \\
\hline No. of parameters & 172 \\
\hline No. of restraints & 1 \\
\hline $\mathrm{H}$-atom treatment & H-atom parameters constrained \\
\hline$\Delta \rho_{\max }, \Delta \rho_{\min }\left(\mathrm{e} \AA^{-3}\right)$ & $0.23,-0.22$ \\
\hline Absolute structure & $\begin{array}{l}\text { Flack } x \text { determined using } 1094 \\
\quad \text { quotients }\left[\left(I^{+}\right)-\left(I^{-}\right)\right] /\left[\left(I^{+}\right)+\left(I^{-}\right)\right] \\
\quad \text { (Parsons et al., 2013) }\end{array}$ \\
\hline Absolute structure parameter & $0.07(4)$ \\
\hline
\end{tabular}

Computer programs: CrysAlis PRO (Rigaku OD, 2018), SHELXT (Sheldrick, 2015a), SHELXL (Sheldrick, 2015b) and OLEX2 (Dolomanov et al., 2009).

Al-Hourani, B. J., Sharma, S. K., Suresh, M. \& Wuest, F. (2012). Bioorg. Med. Chem. Lett. 22, 2235-2238.

Alves, J. A. C., Dillon, C. J. \& Johnstone, R. A. W. (1996). Acta Cryst. C52, 3163-3165.

Aromí, G., Barrios, L. A., Roubeau, O. \& Gamez, P. (2011). Coord. Chem. Rev. 255, 485-546.

Bodrov, A. V., Nikitina, L. E., Startseva, V. A., Lodochnikova, O. A., Musin, R. Z. \& Gnezdilov, O. I. (2013). Russ. J. Gen. Chem. 83, 8086.

Dolomanov, O. V., Bourhis, L. J., Gildea, R. J., Howard, J. A. K. \& Puschmann, H. (2009). J. Appl. Cryst. 42, 339-341.

Gaponik, P. N., Voitekhovich, S. V. \& Ivashkevich, O. A. (2006). Russ. Chem. Rev. 75, 507-539.

Groom, C. R., Bruno, I. J., Lightfoot, M. P. \& Ward, S. C. (2016). Acta Cryst. B72, 171-179.

Kim, Y. J., Han, J.-T., Kang, S., Han, W. S. \& Lee, S. W. (2003). Dalton Trans. pp. 3357-3364.

Mackenzie, C. F., Spackman, P. R., Jayatilaka, D. \& Spackman, M. A. (2017). IUCrJ, 4, 575-587.

Ostrovskii, V. A., Popova, E. A. \& Trifonov, R. E. (2017). Advances in Heterocyclic Chemistry, Vol. 123, edited by Eric F. V. Scriven \& Christopher A. Ramsden, ch. 1, Developments in Tetrazole Chemistry (2009-16), pp. 1-62. New York: Academic Press.

Parsons, S., Flack, H. D. \& Wagner, T. (2013). Acta Cryst. B69, 249259.

Pegklidou, K., Koukoulitsa, C., Nicolaou, I. \& Demopoulos, V. (2010). Bioorg. Med. Chem. 18, 2107-2114.

Pokhodylo, N. T., Shyyka, O. Ya., Matiychuk, V. S. \& Obushak, M. D. (2015). ACS Comb. Sci. 17, 399-403. 
Rigaku OD (2018). CrysAlis PRO. Rigaku Oxford Diffraction, Yarnton, England.

Sheldrick, G. M. (2015a). Acta Cryst. A71, 3-8.

Sheldrick, G. M. (2015b). Acta Cryst. C71, 3-8.

Shyyka, O. Ya., Pokhodylo, N. T., Slyvka, Yu. I., Goreshnik, E. A. \& Obushak, M. D. (2018). Tetrahedron Lett. 59, 1112-1115.

Slyvka, Yu., Fedorchuk, A. A., Pokhodylo, N. T., Lis, T., Kityk, I. V. \& Mys'kiv, M. G. (2018). Polyhedron, 147, 86-93.

Slyvka, Yu., Goreshnik, E., Veryasov, G., Morozov, D., Fedorchuk, A. A., Pokhodylo, N., Kityk, I. \& Mys'kiv, M. (2019). J. Coord. Chem. 72, 1049-1063.
Slyvka, Yu., Pavlyuk, O., Pokhodylo, N., Ardan, B., Mazej, Z. \& Goreshnik, E. (2011). Acta Chim. Slov. 58, 134-138.

Slyvka, Yu., Pokhodylo, N., Savka, R., Mazej, Z., Mys'kiv, M. \& Goreshnik, E. (2010). Chem. Met. Alloys, 2, 130-137.

Slyvka, Yu. I. (2015). J. Struct. Chem. 56, 998-999.

Spackman, M. A. \& Jayatilaka, D. (2009). CrystEngComm, 11, 19 32.

Turner, M. J., Mckinnon, J. J., Wolff, S. K., Grimwood, D. J., Spackman, P. R., Jayatilaka, D. \& Spackman, M. A. (2017). CrystalExplorer17. The University of Western Australia. 


\section{supporting information}

Acta Cryst. (2019). E75, 1331-1335 [https://doi.org/10.1107/S2056989019011459]

\section{Crystal structure, Hirshfeld surface analysis and computational studies of}

\section{5-[(prop-2-en-1-yl)sulfanyl]-1-[2-(trifluoromethyl) phenyl]-1H-tetrazole}

\section{Yurii Slyvka, Evgeny Goreshnik, Nazariy Pokhodylo and Marian Mys`kiv}

Computing details

Data collection: CrysAlis PRO (Rigaku OD, 2018); cell refinement: CrysAlis PRO (Rigaku OD, 2018); data reduction: CrysAlis PRO (Rigaku OD, 2018); program(s) used to solve structure: SHELXT (Sheldrick, 2015a); program(s) used to refine structure: SHELXL (Sheldrick, 2015b); molecular graphics: OLEX2 (Dolomanov et al., 2009); software used to prepare material for publication: OLEX2 (Dolomanov et al., 2009).

5-[(Prop-2-en-1-yl)sulfanyl]-1-[2-(trifluoromethyl) phenyl]-1H-tetrazole

Crystal data

$\mathrm{C}_{11} \mathrm{H}_{9} \mathrm{~F}_{3} \mathrm{~N}_{4} \mathrm{~S}$

$M_{r}=286.28$

Orthorhombic, Pna2 $_{1}$

$a=7.6595(3) \AA$

$b=20.9841(7) \AA$

$c=7.8641(3) \AA$

$V=1263.98(8) \AA^{3}$

$Z=4$

$F(000)=584$

Data collection

Rigaku Oxford Diffraction New Gemini, Dual, $\mathrm{Cu}$ at home/near, Atlas diffractometer

Detector resolution: 10.6426 pixels $\mathrm{mm}^{-1}$

$\omega$ scans

Absorption correction: analytical

(CrysAlis PRO; Rigaku OD, 2018)

$T_{\min }=0.929, T_{\max }=0.969$

Refinement

Refinement on $F^{2}$

Least-squares matrix: full

$R\left[F^{2}>2 \sigma\left(F^{2}\right)\right]=0.035$

$w R\left(F^{2}\right)=0.079$

$S=1.07$

3094 reflections

172 parameters

1 restraint

Hydrogen site location: inferred from neighbouring sites
$D_{\mathrm{x}}=1.504 \mathrm{Mg} \mathrm{m}^{-3}$

Mo $K \alpha$ radiation, $\lambda=0.71073 \AA$

Cell parameters from 9726 reflections

$\theta=3.9-28.4^{\circ}$

$\mu=0.28 \mathrm{~mm}^{-1}$

$T=150 \mathrm{~K}$

Block, colourless

$0.35 \times 0.24 \times 0.15 \mathrm{~mm}$

28170 measured reflections

3094 independent reflections

2730 reflections with $I>2 \sigma(I)$

$R_{\text {int }}=0.059$

$\theta_{\max }=28.9^{\circ}, \theta_{\min }=2.8^{\circ}$

$h=-10 \rightarrow 10$

$k=-28 \rightarrow 28$

$l=-10 \rightarrow 10$

$\mathrm{H}$-atom parameters constrained

$w=1 /\left[\sigma^{2}\left(F_{\mathrm{o}}^{2}\right)+(0.0298 P)^{2}+0.4395 P\right]$

where $P=\left(F_{\mathrm{o}}^{2}+2 F_{\mathrm{c}}{ }^{2}\right) / 3$

$(\Delta / \sigma)_{\max }<0.001$

$\Delta \rho_{\max }=0.23$ e $\AA^{-3}$

$\Delta \rho_{\min }=-0.22$ e $\AA^{-3}$

Absolute structure: Flack $x$ determined using 1094 quotients $\left[\left(I^{+}\right)-(I)\right] /\left[\left(I^{+}\right)+\left(I^{-}\right)\right]$(Parsons et al., 2013)

Absolute structure parameter: 0.07 (4) 


\section{Special details}

Geometry. All esds (except the esd in the dihedral angle between two 1.s. planes) are estimated using the full covariance matrix. The cell esds are taken into account individually in the estimation of esds in distances, angles and torsion angles; correlations between esds in cell parameters are only used when they are defined by crystal symmetry. An approximate (isotropic) treatment of cell esds is used for estimating esds involving l.s. planes.

Refinement. 1. Fixed Uiso At 1.2 times of: All $\mathrm{C}(\mathrm{H})$ groups, $\mathrm{All} \mathrm{C}(\mathrm{H}, \mathrm{H})$ groups 2.a Secondary $\mathrm{CH} 2$ refined with riding coordinates: C2(H2A,H2B) 2.b Aromatic/amide H refined with riding coordinates: C3(H3), C6(H6), C7(H7), C8(H8), C9(H9) 2.c X=CH2 refined with riding coordinates: $\mathrm{C} 4(\mathrm{H} 4 \mathrm{~A}, \mathrm{H} 4 \mathrm{~B})$

Fractional atomic coordinates and isotropic or equivalent isotropic displacement parameters $\left(\AA^{2}\right)$

\begin{tabular}{|c|c|c|c|c|}
\hline & $x$ & $y$ & $z$ & $U_{\text {iso }} * / U_{\text {eq }}$ \\
\hline S1 & $0.82933(7)$ & $0.43951(3)$ & 0.47114 (11) & $0.02848(15)$ \\
\hline F1 & $0.4353(3)$ & $0.24877(9)$ & $0.1989(3)$ & 0.0619 (6) \\
\hline $\mathrm{F} 2$ & $0.5490(3)$ & 0.34169 (11) & $0.2020(2)$ & $0.0590(6)$ \\
\hline F3 & $0.2811(3)$ & $0.32951(12)$ & $0.2643(3)$ & $0.0614(6)$ \\
\hline N1 & $0.4878(3)$ & $0.41022(10)$ & $0.5110(3)$ & $0.0263(5)$ \\
\hline $\mathrm{N} 2$ & 0.3257 (3) & $0.43591(10)$ & $0.4866(5)$ & $0.0357(6)$ \\
\hline N3 & $0.3518(3)$ & 0.49149 (11) & $0.4245(4)$ & $0.0406(7)$ \\
\hline N4 & $0.5253(3)$ & 0.50465 (11) & 0.4058 (3) & $0.0348(6)$ \\
\hline $\mathrm{C} 1$ & $0.6066(3)$ & $0.45334(10)$ & $0.4616(4)$ & $0.0252(5)$ \\
\hline $\mathrm{C} 2$ & 0.9068 (3) & $0.51253(12)$ & $0.3701(3)$ & $0.0264(5)$ \\
\hline $\mathrm{H} 2 \mathrm{~A}$ & 1.030061 & 0.506647 & 0.335573 & $0.032 *$ \\
\hline $\mathrm{H} 2 \mathrm{~B}$ & 0.837482 & 0.520356 & 0.265975 & $0.032 *$ \\
\hline $\mathrm{C} 3$ & $0.8943(3)$ & $0.56925(10)$ & $0.4825(5)$ & $0.0301(5)$ \\
\hline H3 & 0.781849 & 0.582487 & 0.519235 & $0.036^{*}$ \\
\hline $\mathrm{C} 4$ & 1.0299 (4) & 0.60194 (13) & $0.5336(4)$ & $0.0378(7)$ \\
\hline $\mathrm{H} 4 \mathrm{~A}$ & 1.143885 & 0.589750 & 0.498837 & $0.045^{*}$ \\
\hline $\mathrm{H} 4 \mathrm{~B}$ & 1.014415 & 0.637872 & 0.605561 & $0.045^{*}$ \\
\hline $\mathrm{C} 5$ & $0.5129(3)$ & 0.34687 (11) & $0.5739(3)$ & $0.0253(5)$ \\
\hline C6 & 0.5647 (4) & $0.33874(16)$ & 0.7405 (4) & $0.0363(7)$ \\
\hline H6 & 0.580710 & 0.374616 & 0.812478 & $0.044^{*}$ \\
\hline $\mathrm{C} 7$ & $0.5933(5)$ & $0.27776(17)$ & 0.8017 (4) & $0.0436(8)$ \\
\hline H7 & 0.631875 & 0.271828 & 0.915363 & $0.052 *$ \\
\hline $\mathrm{C} 8$ & $0.5662(4)$ & $0.22597(15)$ & $0.6992(5)$ & $0.0427(8)$ \\
\hline H8 & 0.583610 & 0.184288 & 0.743224 & $0.051^{*}$ \\
\hline C9 & $0.5136(4)$ & $0.23375(13)$ & $0.5317(4)$ & $0.0370(7)$ \\
\hline H9 & 0.495146 & 0.197514 & 0.461602 & $0.044 *$ \\
\hline $\mathrm{C} 10$ & 0.4879 (3) & $0.29472(11)$ & 0.4665 (4) & $0.0278(5)$ \\
\hline C11 & $0.4394(4)$ & $0.30342(14)$ & $0.2845(4)$ & $0.0373(7)$ \\
\hline
\end{tabular}

Atomic displacement parameters $\left(\AA^{2}\right)$

\begin{tabular}{lllllll}
\hline & $U^{11}$ & $U^{22}$ & $U^{33}$ & $U^{12}$ & $U^{13}$ & $U^{23}$ \\
\hline S1 & $0.0220(3)$ & $0.0207(2)$ & $0.0428(3)$ & $0.0028(2)$ & $-0.0018(3)$ & $0.0025(4)$ \\
F1 & $0.1032(19)$ & $0.0361(10)$ & $0.0464(12)$ & $-0.0050(11)$ & $-0.0153(12)$ & $-0.0163(10)$ \\
F2 & $0.0844(16)$ & $0.0638(14)$ & $0.0287(10)$ & $-0.0314(12)$ & $0.0085(10)$ & $0.0003(10)$ \\
F3 & $0.0620(13)$ & $0.0750(15)$ & $0.0472(11)$ & $0.0155(11)$ & $-0.0163(10)$ & $-0.0005(11)$
\end{tabular}




\begin{tabular}{lllllll} 
N1 & $0.0222(10)$ & $0.0226(10)$ & $0.0341(14)$ & $0.0019(8)$ & $0.0027(9)$ & $-0.0039(9)$ \\
N2 & $0.0222(9)$ & $0.0327(11)$ & $0.0522(16)$ & $0.0065(8)$ & $0.0027(12)$ & $-0.0053(13)$ \\
N3 & $0.0282(12)$ & $0.0328(13)$ & $0.0607(19)$ & $0.0074(9)$ & $-0.0022(11)$ & $-0.0004(11)$ \\
N4 & $0.0269(12)$ & $0.0265(11)$ & $0.0511(15)$ & $0.0058(9)$ & $-0.0015(11)$ & $0.0045(11)$ \\
C1 & $0.0223(10)$ & $0.0218(10)$ & $0.0317(13)$ & $0.0029(8)$ & $0.0014(14)$ & $-0.0030(13)$ \\
C2 & $0.0260(13)$ & $0.0243(13)$ & $0.0290(14)$ & $-0.0003(10)$ & $0.0015(11)$ & $0.0011(10)$ \\
C3 & $0.0306(12)$ & $0.0228(11)$ & $0.0368(14)$ & $0.0022(9)$ & $0.0041(15)$ & $0.0025(14)$ \\
C4 & $0.0427(16)$ & $0.0251(13)$ & $0.0456(18)$ & $-0.0011(12)$ & $0.0008(13)$ & $-0.0017(12)$ \\
C5 & $0.0232(12)$ & $0.0229(12)$ & $0.0297(14)$ & $-0.0001(10)$ & $0.0053(11)$ & $0.0001(11)$ \\
C6 & $0.0404(17)$ & $0.0397(17)$ & $0.0288(16)$ & $0.0005(14)$ & $0.0036(13)$ & $-0.0043(13)$ \\
C7 & $0.049(2)$ & $0.051(2)$ & $0.0311(16)$ & $0.0076(15)$ & $0.0049(14)$ & $0.0108(14)$ \\
C8 & $0.0466(18)$ & $0.0338(15)$ & $0.0477(19)$ & $0.0052(13)$ & $0.0142(15)$ & $0.0146(15)$ \\
C9 & $0.0415(16)$ & $0.0257(13)$ & $0.0440(17)$ & $-0.0033(12)$ & $0.0099(13)$ & $0.0025(12)$ \\
C10 & $0.0279(11)$ & $0.0237(11)$ & $0.0318(13)$ & $-0.0034(9)$ & $0.0055(14)$ & $-0.0005(13)$ \\
C11 & $0.0489(19)$ & $0.0294(15)$ & $0.0337(16)$ & $-0.0053(13)$ & $-0.0017(13)$ & $-0.0046(12)$ \\
& & & & & & \\
\hline
\end{tabular}

Geometric parameters $\left(\AA,{ }^{\circ}\right)$

\begin{tabular}{|c|c|c|c|}
\hline $\mathrm{S} 1-\mathrm{C} 1$ & $1.732(2)$ & $\mathrm{C} 3-\mathrm{C} 4$ & $1.308(4)$ \\
\hline $\mathrm{S} 1-\mathrm{C} 2$ & $1.825(3)$ & $\mathrm{C} 4-\mathrm{H} 4 \mathrm{~A}$ & 0.9500 \\
\hline $\mathrm{F} 1-\mathrm{C} 11$ & $1.330(3)$ & $\mathrm{C} 4-\mathrm{H} 4 \mathrm{~B}$ & 0.9500 \\
\hline $\mathrm{F} 2-\mathrm{C} 11$ & $1.331(4)$ & $\mathrm{C} 5-\mathrm{C} 6$ & $1.380(4)$ \\
\hline $\mathrm{F} 3-\mathrm{C} 11$ & $1.340(4)$ & $\mathrm{C} 5-\mathrm{C} 10$ & $1.396(4)$ \\
\hline $\mathrm{N} 1-\mathrm{N} 2$ & $1.367(3)$ & C6- $\mathrm{H} 6$ & 0.9500 \\
\hline $\mathrm{N} 1-\mathrm{C} 1$ & $1.341(3)$ & $\mathrm{C} 6-\mathrm{C} 7$ & $1.384(5)$ \\
\hline N1-C5 & $1.431(3)$ & $\mathrm{C} 7-\mathrm{H} 7$ & 0.9500 \\
\hline $\mathrm{N} 2-\mathrm{N} 3$ & $1.280(3)$ & $\mathrm{C} 7-\mathrm{C} 8$ & $1.369(5)$ \\
\hline $\mathrm{N} 3-\mathrm{N} 4$ & $1.365(3)$ & $\mathrm{C} 8-\mathrm{H} 8$ & 0.9500 \\
\hline $\mathrm{N} 4-\mathrm{C} 1$ & $1.319(3)$ & $\mathrm{C} 8-\mathrm{C} 9$ & $1.387(5)$ \\
\hline $\mathrm{C} 2-\mathrm{H} 2 \mathrm{~A}$ & 0.9900 & C9-H9 & 0.9500 \\
\hline $\mathrm{C} 2-\mathrm{H} 2 \mathrm{~B}$ & 0.9900 & $\mathrm{C} 9-\mathrm{C} 10$ & $1.392(4)$ \\
\hline $\mathrm{C} 2-\mathrm{C} 3$ & $1.486(4)$ & $\mathrm{C} 10-\mathrm{C} 11$ & $1.490(4)$ \\
\hline $\mathrm{C} 3-\mathrm{H} 3$ & 0.9500 & & \\
\hline $\mathrm{C} 1-\mathrm{S} 1-\mathrm{C} 2$ & 99.24 (12) & $\mathrm{C} 6-\mathrm{C} 5-\mathrm{C} 10$ & $121.1(2)$ \\
\hline $\mathrm{N} 2-\mathrm{N} 1-\mathrm{C} 5$ & $122.5(2)$ & $\mathrm{C} 10-\mathrm{C} 5-\mathrm{N} 1$ & $120.0(2)$ \\
\hline $\mathrm{C} 1-\mathrm{N} 1-\mathrm{N} 2$ & $108.0(2)$ & $\mathrm{C} 5-\mathrm{C} 6-\mathrm{H} 6$ & 120.3 \\
\hline $\mathrm{C} 1-\mathrm{N} 1-\mathrm{C} 5$ & $129.5(2)$ & $\mathrm{C} 5-\mathrm{C} 6-\mathrm{C} 7$ & $119.3(3)$ \\
\hline $\mathrm{N} 3-\mathrm{N} 2-\mathrm{N} 1$ & $105.7(2)$ & $\mathrm{C} 7-\mathrm{C} 6-\mathrm{H} 6$ & 120.3 \\
\hline $\mathrm{N} 2-\mathrm{N} 3-\mathrm{N} 4$ & $112.2(2)$ & $\mathrm{C} 6-\mathrm{C} 7-\mathrm{H} 7$ & 119.8 \\
\hline $\mathrm{C} 1-\mathrm{N} 4-\mathrm{N} 3$ & $105.0(2)$ & $\mathrm{C} 8-\mathrm{C} 7-\mathrm{C} 6$ & $120.4(3)$ \\
\hline $\mathrm{N} 1-\mathrm{C} 1-\mathrm{S} 1$ & $122.86(17)$ & $\mathrm{C} 8-\mathrm{C} 7-\mathrm{H} 7$ & 119.8 \\
\hline $\mathrm{N} 4-\mathrm{C} 1-\mathrm{S} 1$ & $128.06(19)$ & $\mathrm{C} 7-\mathrm{C} 8-\mathrm{H} 8$ & 119.7 \\
\hline $\mathrm{N} 4-\mathrm{C} 1-\mathrm{N} 1$ & $109.1(2)$ & $\mathrm{C} 7-\mathrm{C} 8-\mathrm{C} 9$ & $120.6(3)$ \\
\hline $\mathrm{S} 1-\mathrm{C} 2-\mathrm{H} 2 \mathrm{~A}$ & 109.0 & $\mathrm{C} 9-\mathrm{C} 8-\mathrm{H} 8$ & 119.7 \\
\hline $\mathrm{S} 1-\mathrm{C} 2-\mathrm{H} 2 \mathrm{~B}$ & 109.0 & $\mathrm{C} 8-\mathrm{C} 9-\mathrm{H} 9$ & 120.0 \\
\hline $\mathrm{H} 2 \mathrm{~A}-\mathrm{C} 2-\mathrm{H} 2 \mathrm{~B}$ & 107.8 & $\mathrm{C} 8-\mathrm{C} 9-\mathrm{C} 10$ & $120.0(3)$ \\
\hline $\mathrm{C} 3-\mathrm{C} 2-\mathrm{S} 1$ & 113.13 (19) & $\mathrm{C} 10-\mathrm{C} 9-\mathrm{H} 9$ & 120.0 \\
\hline
\end{tabular}




\begin{tabular}{|c|c|c|c|}
\hline $\mathrm{C} 3-\mathrm{C} 2-\mathrm{H} 2 \mathrm{~A}$ & 109.0 & $\mathrm{C} 5-\mathrm{C} 10-\mathrm{C} 11$ & $121.3(2)$ \\
\hline $\mathrm{C} 3-\mathrm{C} 2-\mathrm{H} 2 \mathrm{~B}$ & 109.0 & $\mathrm{C} 9-\mathrm{C} 10-\mathrm{C} 5$ & $118.5(3)$ \\
\hline $\mathrm{C} 2-\mathrm{C} 3-\mathrm{H} 3$ & 118.3 & $\mathrm{C} 9-\mathrm{C} 10-\mathrm{C} 11$ & $120.1(3)$ \\
\hline $\mathrm{C} 4-\mathrm{C} 3-\mathrm{C} 2$ & $123.5(2)$ & $\mathrm{F} 1-\mathrm{C} 11-\mathrm{F} 2$ & $106.8(3)$ \\
\hline $\mathrm{C} 4-\mathrm{C} 3-\mathrm{H} 3$ & 118.3 & $\mathrm{~F} 1-\mathrm{C} 11-\mathrm{F} 3$ & $105.7(3)$ \\
\hline $\mathrm{C} 3-\mathrm{C} 4-\mathrm{H} 4 \mathrm{~A}$ & 120.0 & $\mathrm{~F} 1-\mathrm{C} 11-\mathrm{C} 10$ & $112.7(2)$ \\
\hline $\mathrm{C} 3-\mathrm{C} 4-\mathrm{H} 4 \mathrm{~B}$ & 120.0 & $\mathrm{~F} 2-\mathrm{C} 11-\mathrm{F} 3$ & $105.5(3)$ \\
\hline $\mathrm{H} 4 \mathrm{~A}-\mathrm{C} 4-\mathrm{H} 4 \mathrm{~B}$ & 120.0 & $\mathrm{~F} 2-\mathrm{C} 11-\mathrm{C} 10$ & $112.6(2)$ \\
\hline $\mathrm{C} 6-\mathrm{C} 5-\mathrm{N} 1$ & $118.8(2)$ & $\mathrm{F} 3-\mathrm{C} 11-\mathrm{C} 10$ & $112.9(2)$ \\
\hline $\mathrm{S} 1-\mathrm{C} 2-\mathrm{C} 3-\mathrm{C} 4$ & $117.0(3)$ & $\mathrm{C} 5-\mathrm{N} 1-\mathrm{N} 2-\mathrm{N} 3$ & $-177.7(2)$ \\
\hline $\mathrm{N} 1-\mathrm{N} 2-\mathrm{N} 3-\mathrm{N} 4$ & $0.0(4)$ & $\mathrm{C} 5-\mathrm{N} 1-\mathrm{C} 1-\mathrm{S} 1$ & $-1.9(4)$ \\
\hline $\mathrm{N} 1-\mathrm{C} 5-\mathrm{C} 6-\mathrm{C} 7$ & $-178.5(3)$ & $\mathrm{C} 5-\mathrm{N} 1-\mathrm{C} 1-\mathrm{N} 4$ & $177.3(2)$ \\
\hline $\mathrm{N} 1-\mathrm{C} 5-\mathrm{C} 10-\mathrm{C} 9$ & $179.9(2)$ & $\mathrm{C} 5-\mathrm{C} 6-\mathrm{C} 7-\mathrm{C} 8$ & $-1.6(5)$ \\
\hline $\mathrm{N} 1-\mathrm{C} 5-\mathrm{C} 10-\mathrm{C} 11$ & $1.5(4)$ & $\mathrm{C} 5-\mathrm{C} 10-\mathrm{C} 11-\mathrm{F} 1$ & $174.8(2)$ \\
\hline $\mathrm{N} 2-\mathrm{N} 1-\mathrm{C} 1-\mathrm{S} 1$ & $-179.8(3)$ & $\mathrm{C} 5-\mathrm{C} 10-\mathrm{C} 11-\mathrm{F} 2$ & $53.9(4)$ \\
\hline $\mathrm{N} 2-\mathrm{N} 1-\mathrm{C} 1-\mathrm{N} 4$ & $-0.6(3)$ & $\mathrm{C} 5-\mathrm{C} 10-\mathrm{C} 11-\mathrm{F} 3$ & $-65.4(3)$ \\
\hline $\mathrm{N} 2-\mathrm{N} 1-\mathrm{C} 5-\mathrm{C} 6$ & $-104.5(3)$ & $\mathrm{C} 6-\mathrm{C} 5-\mathrm{C} 10-\mathrm{C} 9$ & $1.1(4)$ \\
\hline $\mathrm{N} 2-\mathrm{N} 1-\mathrm{C} 5-\mathrm{C} 10$ & $76.6(3)$ & $\mathrm{C} 6-\mathrm{C} 5-\mathrm{C} 10-\mathrm{C} 11$ & $-177.3(3)$ \\
\hline $\mathrm{N} 2-\mathrm{N} 3-\mathrm{N} 4-\mathrm{C} 1$ & $-0.3(4)$ & $\mathrm{C} 6-\mathrm{C} 7-\mathrm{C} 8-\mathrm{C} 9$ & $1.4(5)$ \\
\hline $\mathrm{N} 3-\mathrm{N} 4-\mathrm{C} 1-\mathrm{S} 1$ & $179.7(2)$ & $\mathrm{C} 7-\mathrm{C} 8-\mathrm{C} 9-\mathrm{C} 10$ & $0.0(5)$ \\
\hline $\mathrm{N} 3-\mathrm{N} 4-\mathrm{C} 1-\mathrm{N} 1$ & $0.6(3)$ & $\mathrm{C} 8-\mathrm{C} 9-\mathrm{C} 10-\mathrm{C} 5$ & $-1.2(4)$ \\
\hline $\mathrm{C} 1-\mathrm{S} 1-\mathrm{C} 2-\mathrm{C} 3$ & $75.0(2)$ & $\mathrm{C} 8-\mathrm{C} 9-\mathrm{C} 10-\mathrm{C} 11$ & $177.2(3)$ \\
\hline $\mathrm{C} 1-\mathrm{N} 1-\mathrm{N} 2-\mathrm{N} 3$ & $0.4(4)$ & $\mathrm{C} 9-\mathrm{C} 10-\mathrm{C} 11-\mathrm{F} 1$ & $-3.6(4)$ \\
\hline $\mathrm{C} 1-\mathrm{N} 1-\mathrm{C} 5-\mathrm{C} 6$ & $77.8(4)$ & $\mathrm{C} 9-\mathrm{C} 10-\mathrm{C} 11-\mathrm{F} 2$ & $-124.5(3)$ \\
\hline $\mathrm{C} 1-\mathrm{N} 1-\mathrm{C} 5-\mathrm{C} 10$ & $-101.1(3)$ & $\mathrm{C} 9-\mathrm{C} 10-\mathrm{C} 11-\mathrm{F} 3$ & $116.2(3)$ \\
\hline $\mathrm{C} 2-\mathrm{S} 1-\mathrm{C} 1-\mathrm{N} 1$ & $175.3(2)$ & $\mathrm{C} 10-\mathrm{C} 5-\mathrm{C} 6-\mathrm{C} 7$ & $0.3(4)$ \\
\hline $\mathrm{C} 2-\mathrm{S} 1-\mathrm{C} 1-\mathrm{N} 4$ & $-3.8(3)$ & & \\
\hline
\end{tabular}

Hydrogen-bond geometry $\left(\AA,{ }^{\circ}\right)$

\begin{tabular}{lllll}
\hline$D-\mathrm{H} \cdots A$ & $D-\mathrm{H}$ & $\mathrm{H} \cdots A$ & $D \cdots A$ & $D-\mathrm{H} \cdots A$ \\
\hline $\mathrm{C} 2-\mathrm{H} 2 A \cdots \mathrm{N} 3^{\mathrm{i}}$ & 0.99 & 2.58 & $3.464(3)$ & 148 \\
$\mathrm{C} 2-\mathrm{H} 2 B \cdots \mathrm{N} 2^{\mathrm{ii}}$ & 0.99 & 2.69 & $3.666(4)$ & 169 \\
$\mathrm{C} 3-\mathrm{H} 3 \cdots \mathrm{F}^{3 i i}$ & 0.95 & 2.71 & $3.351(3)$ & 125 \\
$\mathrm{C} 4-\mathrm{H} 4 A \cdots \mathrm{N} 3^{\mathrm{i}}$ & 0.95 & 2.67 & $3.491(3)$ & 145 \\
$\mathrm{C} 4-\mathrm{H} 4 B \cdots \mathrm{F} 1^{\mathrm{iv}}$ & 0.95 & 2.47 & $3.355(3)$ & 155 \\
$\mathrm{C} 6-\mathrm{H} 6 \cdots \mathrm{N} 4^{i i i}$ & 0.95 & 2.76 & $3.601(3)$ & 148
\end{tabular}

Symmetry codes: (i) $x+1, y, z$; (ii) $-x+1,-y+1, z-1 / 2$; (iii) $-x+1,-y+1, z+1 / 2$; (iv) $-x+3 / 2, y+1 / 2, z+1 / 2$. 